\title{
FASES DE LA METODOLOGÍA DE LA INVESTIGACIÓN LA LLAVE PARA ABRIR CAMINO EN LA SELVA DE LOS HECHOS*
}

\author{
Raúl Omar Di Marco Morales \\ Magíster en Administración de Empresas \\ Universidad Santo Tomás, Bucaramanga \\ raulomard@yahoo.com
}

\begin{abstract}
Resumen
El artículo muestra las etapas para el desarrollo de una investigación científica desde la formulación del problema, la construcción de un marco teórico que demuestre el sentido de los datos que son requeridos para satisfacer las hipótesis planteadas y organizándolos conforme a una estructura lógica: la Matriz de Datos; luego se procede a utilizar dentro del método científico la lógica en su expresión hipotético-deductivo, para llegar a un producto netamente científico.
\end{abstract}

Palabras Clave: Fases, Metodología, Investigación, Ciencia, Matriz de Datos, Proceso científico, Inferencia, Hipótesis.

\begin{abstract}
This article shows the steps for the development of scientific research from the formulation of the problem by building a theoretical framework that shows the meaning of the data that are required to satisfy the hypotheses and organizing them according to a logical structure: the data matrix; then proceeds to use within the logic of the scientific method in their expression hypothetical deductive, to reach a product purely scientific.
\end{abstract}

Key words: Phases, Methodology, Research, Science, Data Matrix, Scientific process, Inference, Hypothesis.

\section{Introducción}

Una investigación consiste en una serie de actividades que se deben desarrollar dentro de proceso metódico, como son: el planteamiento del problema, la formulación de hipótesis, hasta la construcción de la matriz de datos, de las características y propósitos que se persiguen, que finalmente han de dejar un material que servirá como materia prima para nuevas investigaciones; por tal motivo, queda de acuerdo con lo que dice Samaja (2010), cuando indica que el "proceso de investigación se refiere a la totalidad de los componentes, dimensiones y momentos de la investigación científica".

El conocer y el saber algo, indica Sabino (1982) que es un problema que radica fundamentalmente en los seres humanos que requieren para desarrollar su vida y responder a sus inquietudes; pero, por otro lado, el mismo Sabino afirma que la verdad no se muestra como tal, que debe ser buscada e indagada por medio de un trabajo de investigación.

De ahí, que la investigación científica debe ser concebida como un proceso mediante el cual un sujeto que se inserta necesariamente en un cierto contexto, con el tiempo alcanzará un producto (un conocimiento más objetivo y satisfactorio) como resultado de las acciones encaminadas y dirigidas hacia un determinado fin como es el de obtener un conocimiento verificable sobre los hechos que nos rodean, por lo que se sitúa al conocimiento científico según Samaja (p.29) como uno de los modos posibles de conocimiento humano, si es lo que se obtiene como producto de un proceso de investigación científica; Bunge (1958, p.11) indica que el conocimiento científico debe ser: fáctico (pues parte de los hechos, descarta hechos, produce nuevos hechos y los explica), ya que la ciencia intenta describir los hechos tales como son; además, claro y preciso (resultados claros y libre de vaguedades), comunicable y verificable $y$, finalmente sistemático, general y predictivo. 


\section{Desarrollo}

Antes de comenzar a problematizar alguna cuestión desde el punto de vista del proyecto de investigación y, en especial, a lo que se refiere a la metodología de la investigación, se debe revisar algunas de las diferentes definiciones ya que en ellas se pueden evidenciar conflictos dentro de la misma investigación; por tal motivo, en la construcción del siguiente marco teórico hay que precisar que un proceso de investigación no es más que una continuidad de momentos metodológicos o fases, que son posibles de distinguir a medida que se moldean, dentro de un camino de carácter dinámico y procesal (no lineal) que deberá recorrer el investigador, de modo tal que no se conciba al proceso mismo con un elemento inicial y con uno final completamente delimitados, sino que se vea como un continuo trabajo donde cada investigación particular es parte de un esfuerzo mucho mayor en el desarrollo de los conocimientos científicos. Ynoub (Inédito I, p. 32) menciona que el desarrollo de un proceso de investigación es un movimiento que se realiza en un ciclo de tres grandes fases, a saber:
Fase I, Sincrética (o ideatoria): donde se precisa tanto conceptual como teóricamente el objetivo de la investigación, ya que parte desde intuiciones muy preliminares que luego se convierten en genuinas conceptualizaciones; posee un núcleo central compuesto de problemas y de hipótesis, flanqueados por un lado por los marcos conceptuales y las tradiciones teóricas de referencia y, por el otro lado, por los objetivos ó productos que se esperan alcanzar. Un problema lo define Ynoub (Inédito I, p. 36), como "una laguna cognitiva" de un tema elegido y que se convierte en preguntas que vienen a organizar toda la investigación (se deriva de la reflexión teórica), mientas las hipótesis son las respuestas presuntivas o tentativas a los problemas que son la interfaz con la siguiente fase; en esta etapa como lo indica Ynoub, los problemas y las hipótesis encierran los núcleos directivos del trabajo y de ellos se deriva toda la investigación; sin embargo, se puede volver a ellos para revisarlos y precisarlos a medida que avanza el trabajo de investigación, debido a que marcan el norte de la búsqueda investigativa, como se observa en el Cuadro 1, ya que tienen una función organizadora en el diseño y el desarrollo de la investigación.

Cuadro 1: El proceso de Investigación

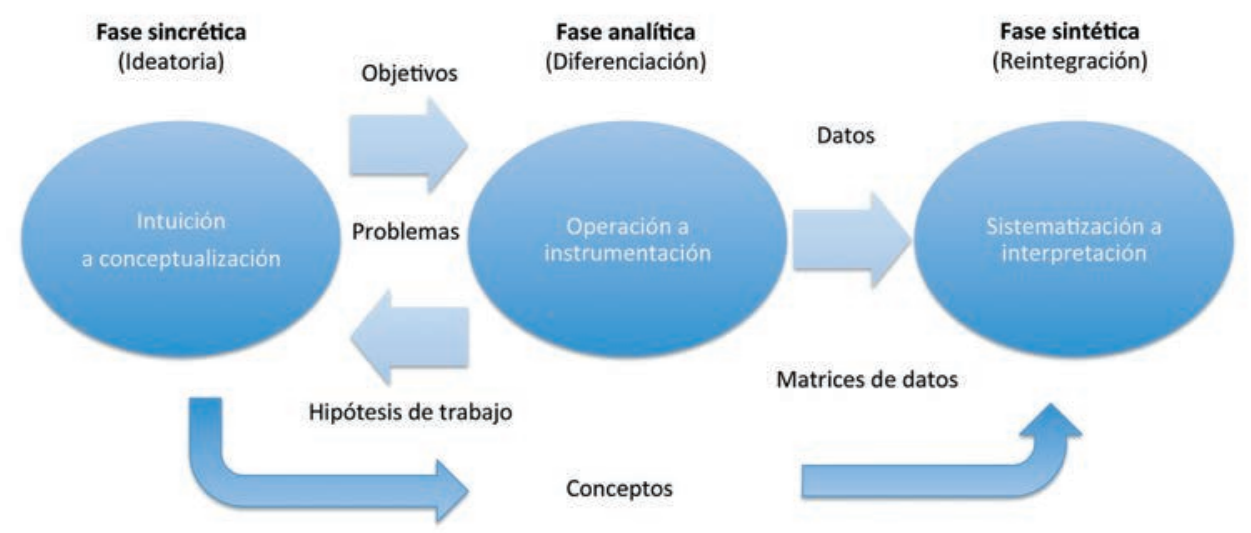

Fuente: YNOUB, R (Inédito I, p. 43)

Fase II: Analítica (o de diferenciación): aquí se especificará la naturaleza de los datos que se desean obtener y el modo de obtenerlos, ya que se pasa del estado anterior (de conceptua- lización) a uno de operacionalización, y que, finalmente llega en el momento de producir los datos, a la instrumentación; Ynoub indica que se identifican y diferencian los componentes 
en que se desagrega el objeto de la investigación y las relaciones que se postulan entre ellos, como las estrategias para su abordaje empírico; de esta manera, la información producida conforma la interfaz con la siguiente fase como se observa en el Cuadro 1.

Fase III: Sintética (o de reintegración): donde se pasa de la producción a la interpretación de datos, con la que se cierra el ciclo del proceso de investigación. De igual forma, como se ve en el Cuadro 1, se tratan los datos para que sean abordables e interpretables en el marco de la teoría, lo que permitirá iluminar las hipótesis y responder a los problemas, o abrir simplemente a nuevos modos de precisarlos con respecto al tema abordado. En esta fase, menciona Ynoub, se sintetizan los componentes teóricos (fase sincrética) con los hallazgos empíricos (fase analítica), por lo que arroja una nueva concepción del objeto de investigación.

Ynoub (Inédito I, p.34) confirma que con mucha frecuencia los hallazgos que se presentan "pueden inspirar nuevos interrogantes", por lo que hay que buscar nuevas respuestas ya que toda interpretación de datos puede ofrecer nuevos caminos y nuevas lecturas, ya que da paso a nuevos objetivos e hipótesis a los que se les debe dar respuesta; de esta manera, el conocimiento o la nueva interpretación sobre algún tema, abre nuevas preguntas y nuevas conjeturas, las que llevan a cimentar las bases para su abordaje e inicio de un nuevo ciclo de investigación; además, se comparte la afirmación de Samaja (2010) cuando expresa que un conocimiento que egresa del proceso de investigación, inmediatamente se transforma en condiciones de investigación para nuevos procesos, y si no es incorporado se vuelve alimento de roedores e insectos y no en un hecho de la cultura científica.

Ya iniciadas labores, se procede a precisar el objeto o tema que se va a investigar, momento en el cual aparece como un desafío la formulación del problema de investigación; pero no es nada fácil, motivo por el cual, Ynoub (Inédito
III) p.13 identifica algunos errores frecuentes en la formulación de problemas de investigación, como son:

1. Preguntas que implican juicios de valor: ¿Es buena la estructura organizacional de una empresa familiar? Las respuestas a esta pregunta implica alguna presunción o justificación valorativa y no apunta a la producción de un conocimiento.

2. Preguntas de información o falsas preguntas: ¿Cuántas empresas familiares fueron creadas para la apertura económica? La respuesta se encuentra accediendo a la información ya disponible, como lo es el de la Cámara de Comercio.

3. Preguntas que apuntan a la intervención: ¿Qué sistemas de apalancamiento financiero pueden implementarse para desarrollar nuevos proyectos de inversión en empresas familiares? La respuesta a esta pregunta es una acción o un plan de acción que apunta a cuestiones de implementación y no de un nuevo conocimiento.

4. Preguntas filosóficas: ¿Qué es la familia? Sólo se trata de un ejercicio de tipo reflexivo, intelectual o conceptual orientada a la definición y el conocimiento de lo general; mientras que la filosofía aborda objetos ideales, la ciencia se interesa por conocer objetos abordables en el marco de una experiencia posible.

5. Preguntas que contienen supuestos sobre escenarios o situaciones no accesibles, no controlables o no manipulables por la investigación: ¿Cómo se evalúa un crédito bancario por parte del sector financiero, para ser tomado por las empresas familiares del sector comercial? Es posible que la información requerida no sea accesible al investigador, es decir, no existe una viabilidad investigativa. 
Ahora se ha de pasar a las hipótesis; cuando se habla de ellas, Sabino (1980, p. 87) la define como una proposición que describe o explicita algún campo de fenómenos en estudio, que pueden ser o no confirmados por los hechos, mientras que Ynoub (Inédito IV, p. 27) indica que las hipótesis se derivan al asumir una presunción acerca de "lo que hay en el mundo" y de lo que entonces se "puede esperar de él", además de expresar alguna regularidad presunta del fenómeno que quiere estudiarse.

Ynoub identifica los siguientes tipos de hipótesis vinculándolas a cada esquema de investigación:

1. Hipótesis de atribución: las que están orientadas a construir o identificar categorías y sistemas clasificatorios, y se presentan en las investigaciones descriptivas donde se propone precisar regularidades de atributos o propiedades, por lo que se requieren de este tipo de hipótesis cuando se trabaja a un nivel exploratorio. Ej.: "En el $80 \%$ de las empresas familiares del sector comercial, las decisiones en la organización son tomadas por su gestor-fundador".

2. Hipótesis de causación: cuando las hipótesis pasan en términos de causa - efecto, presentándose en las investigaciones de esquemas explicativos, donde se trata de estimar o de identificar relaciones de determinación entre variables. Ej.: "La línea de jerarquía en las empresas familiares del sector comercial, conlleva que las decisiones sean tomadas por el familiar con mayor antigüedad dentro de la organización".

3. Hipótesis hermenéuticas: se presentan en las investigaciones de esquema interpretativo, donde una genuina interpretación implica el paso de la causalidad a la imputación. Ejemplo: "Dentro de una empresa familiar del sector comercial, la toma de decisiones por parte de su gestor - fundador es la manera más clara para retener el poder.

Sin embargo, Ynoub (Inédito IV p.59) también muestra que el proceso de investigación puede describirse (luego de adaptar con modificaciones la propuesta de Samaja), en torno a la siguiente serie de hipótesis:

1. Hipótesis nucleares o sustantivas: las que aparecen en la fase sincrética como una conjetura general y que vienen a constituir la hipótesis nuclear o central de la investigación, a la cual se le pide validez (desde el punto de vista teórico o conceptual) ya que ilumina nuevas relaciones no conocidas o no postuladas previamente.

2. Hipótesis indicadoras e instrumentales: las que aparecen en la fase analítica y suponen siempre decisiones que deberán justificarse, en términos de la adecuación empírica, para contrarrestar las hipótesis de la fase anterior.

3. Hipótesis de integración o significación: las que aparecen en la fase sintética a la luz de los resultados y luego de interpretar los datos, por lo cual ya se dio un veredicto ya sea de apreciación o de valoración de hechos, conllevan a una etapa de validación conclusional.

Conviene brevemente describir el proceso que la investigación ha seguido hasta este momento: se realizó la formulación del problema, que de alguna manera es desafiante ya que se recorre un camino referido a la construcción del marco teórico que nos mostrará el sentido de los datos que es preciso obtener para satisfacer las hipótesis planteadas; aquí aparece la noción de dato científico como la información resultante y estructurada, que no se reduce al de información numérica o cuantitativa; Samaja (p.160) muestra el dato como el producto de la traducción de la experiencia 
espontánea a una descripción científica, este es una construcción compleja que posee una estructura interna compuesta por cuatro componentes:

1. Unidad de análisis (UA): son los mismos elementos, entidades, sujetos y objetos en las que se focaliza la descripción o el análisis en un cierto momento de la investigación y que en términos del enunciado descriptivo se refiere al argumento.

2. Variables (V): o dimensiones de análisis son las que constituyen los aspectos, las características, las propiedades ó los atributos que se han seleccionado, para examinar o estudiar a las unidades de análisis; basados en el enunciado descriptivo se refiere a la función misma.

3. Valores (R): o categorías son las que constituyen los estados particulares que pueden asumir las variables y que coincide con el valor de la función cuando se habla en términos del enunciado descriptivo.

4. Indicadores (I): o definiciones operacionales son las que constituyen las maneras de medir o de evaluar las variables y que en términos del enunciado descriptivo se refiere a las operaciones de que está construida y que permiten calcular el valor de la función; tiene dimensión (el qué se medirá) y un procedimiento (el cómo se medirá).

Ynoub (Inédito VI, p. 8) menciona que Johann Galtung advierte que todo dato se organiza conforme a una estructura lógica invariante, a la que denominó matriz de datos. Samaja (2010, p. 163) amplía el enfoque de Galtung al indicar que la matriz de datos es una estructura invariante y a priori de carácter universal que reconoce los cuatro elementos anteriormente nombrados y postula que todo dato implica un proceso constructivo por referencia.
Samaja (2010) indica además que dentro de la matriz de datos se admite el paso de unos niveles a otros conforme a las variables, lo que conformaría un grupo de matrices: la primera referida a una matriz central ó la matriz de datos propiamente dicha, que Samaja llama "Nivel de anclaje"; la segunda, constituida por los componentes de las unidades de análisis del nivel de anclaje, que Samaja denomina como "Matriz de nivel subunitario", y la tercera, la conformada por los contextos de las unidades de análisis del nivel de anclaje, que Samaja la denomina como "Matriz de nivel supraunitaria".

De igual forma Samaja (2010) comenta que "el proceso de elaboración de indicadores forma parte esencial de estas mediaciones entre matrices de diferentes niveles de integración", por lo que todo dato científico vincula un concepto con un estado de cosas del mundo externo mediante la aplicación de un procedimiento a una o más dimensiones consideradas observables, por lo que aparecen de esta manera dos elementos básicos que conforman el indicador: la dimensión, que es considerada como observable, y el procedimiento, para ejecutar la observación.

Además se dirá que un indicador es válido si cumple con la correspondencia entre los contenidos de la dimensión del indicador y lo que se expresa en la variable; mientras que el procedimiento del indicador es confiable si al aplicarlo no se introducen cambios por el efecto de su aplicación; es así que la validez compromete la dimensión (el qué se evalúa) del indicador, mientras que la confiabilidad se relaciona con los procedimientos (el cómo se evalúa) del indicador.

Samaja (2010, p. 184) como conclusión, indica que: "el proceso de investigación es traducción del objeto de investigación a un sistema de matrices de datos y de un sistema de matrices de datos a la unidad de un modelo (análisis de datos) que reproduzca el compor- 
tamiento del objeto real", por lo que investigar científicamente es construir sistemas de matrices de datos científicos, Ilenarlas y procesarlas e interpretar.

Finalmente, Samaja (Inédito I, p.2) afirma que un rasgo peculiar del método científico es su operación hipotético-deductivo, por lo que encontramos en la lógica una herramienta fundamental para llevar a cabo dicho método; la lógica que es una ciencia normativa no nos enseña qué debemos pensar sino cómo debemos pensar, de modo que se pueda decir que el núcleo mismo de la enseñanza de la lógica es:"la teoría de la inferencia".

La inferencia deductiva indica Samaja (Inédito I, p. 8) es "aquella cuya forma está dada por la afirmación de una Regla (Premisa mayor) y un caso de dicha regla (Premisa menor), y la derivación al caso particular del o los rasgos que la regla enuncia en general (Conclusión)", es decir, la deducción va de un saber general (un conjunto de elementos) a uno particular (subconjunto de elementos de ese conjunto); por otro lado está la inferencia inductiva, que va de lo particular a lo general, en el sentido estricto de pasar de proposiciones de un subconjunto de elementos, a proposiciones de su conjunto base.

Samaja además comenta que estas inferencias son las dos formas que más aparecen en los libros de lógica y metodología; por un lado está la deducción, que es caracterizada como esa forma de derivación que cuando sus premisas son verdaderas, la conclusión también lo es (necesariamente), y por el otro lado, la inducción es aquella inferencia que aunque todas sus premisas sean verdaderas, su conclusión queda formalmente indeterminada e inclusive sea falsa, pero si sus premisas son falsas, la conclusión deberá ser necesariamente falsa; Samaja comenta que ambas formas de inferencias parecieran disponer de virtudes lógicas, al lado de vicios formales insuperables.

Una hipótesis que resulte confirmada por las predicciones empíricamente contrastables, podrá ser considerada una buena hipótesis y su uso será válido, pero si no, por el modus tollens deberá ser abandonada; Ynoub (Inédito IV, p.46) menciona que lo único que se puede probar (aunque suene contrariedad) es que la hipótesis es falsa; sin embargo, la propuesta metodológica popperiana se conoce con el nombre de método deductivo, donde se parte de una hipótesis y que a partir de ella se derivan deductivamente la hipótesis de trabajo y los enunciados observacionales. Pese a postular la primacía de la deducción, (Popper 1980:193) reconoce que el contragolpe falsador se comporta como si fuera un procedimiento inductivo.

La ciencia es un método que permite descartar malas teorías, aunque no se nos permita demostrar la verdad, pero cuando se está involucrado un proceso de falsación y sus cualidades lógicas son tenidas en cuenta al momento de dar un veredicto final sobre la solución, se procede a un tipo diferente de inferencia llamada abducción, la cual opera de manera diferente tanto a la deducción como a la inducción para que el sistema mismo de las inferencias no se vuelva paradójico. La abducción es conocida como el razonamiento desde el consecuente al antecedente.

Como se observa en el Cuadro 2, la abducción es muy diferente a la deducción y a la inducción, porque ella no supone un movimiento entre lo general y lo particular, sino un movimiento entre el rasgo $[r]$ y el tipo de objeto [C], a partir de conocer la Regla [R] que relaciona los atributos, indicando que la abducción va de la Parte al Todo, pero no por generalización sino como identificación de la unidad de un proceso.

Los ejemplos para cada tipo de inferencia son:

1. Deducción: Si las empresas son fundadas por familias, y Ford, Walmart, Cargill y Motorola son empresas, entonces Ford, Walmart, Cargill y Motorola son fundadas por familias. 
2. Inducción: Si Ford, Walmart, Cargill y Motorola son empresas, y Ford, Walmart, Cargill y Motorola son fundadas por familias, entonces las empresas son fundadas por familias.

3. Abducción: Si Ford es fundada por una familia, y las empresas son fundadas por familias, entonces Ford es una empresa.

Cuadro 2: Diferentes tipos de inferencia

\begin{tabular}{|c|c|c|}
\hline Deducción & Inducción & Abducción \\
\hline $\begin{array}{c}\text { Conjunto y } \\
\text { Subconjunto }\end{array}$ & $\begin{array}{c}\text { Subconjunto y } \\
\text { Conjunto }\end{array}$ & $\begin{array}{c}\text { Parte-Órgano } \\
\text { Todo-Organismo }\end{array}$ \\
\hline $\mathrm{R}+\mathrm{C} \rightarrow \mathrm{r}$ & $\mathrm{C}+\mathrm{r} \rightarrow \mathrm{R}$ & $\mathrm{r}+\mathrm{R} \rightarrow \mathrm{C}$ \\
\hline \multicolumn{3}{|c|}{ Donde R: Regla C: Caso $\mathrm{r}:$ Rasgo } \\
\hline
\end{tabular}

Fuente: análisis del autor

Por otro lado, se podría caracterizar la inferencia por analogía, la cual se lleva a cabo cuando se tiene como premisa la proposición que afirma que el rasgo que se plantea es similar a otro rasgo de un caso de otro fenómeno; esta otra inferencia da paso a una percepción de una semejanza que nos impulsa a derivar nuestro rasgo [r] que se explica por una Regla [R] semejante a la de nuestro caso, por lo que determina las condiciones de posibilidad de la hipótesis, y que podría describirse de la siguiente manera:

rasgo $[r]+$ Regla [R] Hipotética $\rightarrow$ Caso [C] identificado.

Un ejemplo de una inferencia por analogía, sería:

- Paso 1: Ford es fundada en 1900, entonces es una Empresa legal.

- Paso 2: Ford es fundada por una familia, y constituida para que dure, entonces es una empresa.

- Paso final: Ford es fundada por una familia, y las empresas son fundadas por familias, entonces Ford es una empresa.
Bunge (1958) se refiere a que la deducción, la inducción y la analogía de suposiciones, proveen puntos de partida que deben ser elaborados y probados, ya que la investigación no es errática sino metódica, basada en que en la ciencia no hay caminos reales y que se abre camino en la selva de los hechos.

\section{Conclusiones}

Cuando se habla de Metodología de la Investigación, muchas personas no entienden su importancia y su verdadero accionar para ser aplicado dentro de un proceso investigativo racional, y mucho menos de los beneficios tanto personal como organizacional que se tienen debido a su debida aplicación. Es así que para mí la Metodología de la Investigación es un esfuerzo de reflexión sobre el comportamiento humano-racional que nos educa y que finalmente nos ayuda a conocer, a saber algo acerca de... en busca de satisfacer las necesidades de conocimiento de los individuos que siempre andamos en busca de una respuesta y que gracias a un trabajo metodológico indagatorio precedido de deliberaciones racionales, gran parte de estas respuestas pueden ser encontradas o, por lo menos, darán paso a nuevas y futuras investigaciones. Además, la Metodología de la Investigación nos aproxima rápidamente a una herramienta de trabajo científico con toques de historia, política y cultura en general, la cual ha de servir de brújula para que las personas puedan realizar sus futuras investigaciones dentro de unas normas y de un marco conceptual moderno.

Quisiera retomar una definición de ética que presenta Savater (1998) y ajustarla al proceso investigativo, que no solamente se podría definir como un curso de acción; esta quedaría de la siguiente manera:

"Es un proceso irrenunciable e intransferible, en el que no existe castigo, sanción u obligación, y donde cada individuo decide qué es lo que quiere y lo que no quiere según 
su propia convicción, acorde a la metodología directa y rigurosa, que consolida la base teórica que fundamenta la investigación y la inferencia lógica que respeta las normas establecidas por los filósofos de la ciencia".

Somos profesionales que se nos caracteriza por tener ocupaciones de alta relevancia social, razón por la cual requerimos de una preparación rigurosa y metodológica para cumplir con una investigación científica; sin embargo, un profesional es un ser humano que no está exento de una eventual incompetencia o incapacidad de resolver o afrontar adecuadamente los problemas de la investigación que se le presentan, poniendo en riesgo de alguna forma dicha investigación, lo que puede ser evaluado en términos de fracaso por la comunidad científica.

Finalmente: ¡Qué nos depara el futuro! E independiente de éste ya que no somos adivinos, se debe hablar y apostarle a una conducción por medio del cual se adquieren conocimientos objetivos del mundo. Considero que la metodología de la investigación es el espacio en el cual el investigador encuentra orientaciones que le evitarán vivencias equívocas e innecesarias, ya que la metodología induce a explorar cuidadosamente en el campo donde se pretende actuar, delimitar rigurosamente el objetivo de la investigación, consolidar la bases teórica y conceptual bajo las cuales se realizará la investigación, determinar las diferentes hipótesis y seguir con un proceso de inferencia lógica, para lograr un producto científico.

\section{Referencias Bibliográficas}

Bunge, M. (1958). La ciencia su método y su filosofía. Buenos Aíres: Universidad de Buenos Aíres.

Popper, K. (1980). La lógica de la investigación científica. Madrid: Editorial Tecnos. En: [http://ebookbrowse. com/la-logica-de-la-investigacion-cientifica-karl-rpopper-pdf-d182345521 Consultado, septiembre 26 de 2012]

Sabino, C. A. (1992). El proceso de investigación. Buenos Aires: Lumen.

Samaja, J. (2010). Epistemología y metodología, elementos para una teoría de la investigación científica. Buenos Aires: Editorial Universitaria de Buenos AíresEudeba.

(2012). Inédito I: El papel de la hipótesis y de las formas de inferencia en el trabajo científico

Savater, F. (1998). La dimensión ética de la empresa. Bogotá: Siglo del Hombre Editores.

Ynoub, R. C. (2012). Inédito I: La ciencia como práctica social. Bases para situar el examen del proceso de investigación científica en sentido pleno.

(2012). Inédito III: Problematizar. El nudo argumental del proceso de investigación

(2012). Inédito IV: Sobre modelos, conjeturas y predicciones en el proceso de la investigación

(2012). Inédito VI: Estructura, génesis y dialéctica en la construcción de datos científicos

(2012). El proyecto y la metodología de la investigación. CENGAGE Learning

\section{Cómo citar este artículo:}

Di Marco, M. R. (2013). Fases de la metodología de la investigación. La llave para abrir el camino en la selva de los hechos. Espiral, Revista de Docencia e Investigación. 2 (2), 69-76 